\title{
Decision making in acute DeBakey I aortic dissection: Balancing extensive arch reconstruction versus mortality
}

\author{
Ibrahim Sultan, MD, and Wilson Y. Szeto, MD
}

\author{
From the Division of Cardiovascular Surgery, University of Pennsylvania Medical Center, Philadelphia, Pa. \\ Disclosures: Authors have nothing to disclose with regard to commercial support. \\ Received for publication Oct 10, 2015; accepted for publication Oct 18, 2015; available ahead of print Nov 21, \\ 2015. \\ Address for reprints: Wilson Y. Szeto, MD, Division of Cardiovascular Surgery, University of Pennsylvania \\ Medical Center, Penn Presbyterian Medical Center, 51 N 39th St, Heart a Vascular Pavilion 2A, Philadelphia, \\ PA 19104 (E-mail: wilson.szeto@uphs.upenn.edu). \\ J Thorac Cardiovasc Surg 2016;151:349-50 \\ $0022-5223 / \$ 36.00$ \\ Copyright (c) 2016 by The American Association for Thoracic Surgery \\ http://dx.doi.org/10.1016/j.jtcvs.2015.10.044
}

Management of the aortic arch in acute DeBakey I aortic dissection remains a clinical challenge. Debate persists regarding whether a less aggressive non-total arch reconstruction (non-TAR) or a total arch reconstruction (TAR) is the optimal surgical reconstruction for these acutely ill patients. Increasingly, in most aortic centers of excellence, the extent of arch reconstruction is determined according to a "tear-oriented" paradigm. In their report in this issue of the Journal, Omura and colleagues ${ }^{1}$ report their results of patients undergoing either TAR or non-TAR for acute DeBakey I aortic dissection according to a decision algorithm that is based on such a tear-oriented paradigm.

Omura and colleagues ${ }^{1}$ are to be commended on their impressive outcomes, particularly for their patients undergoing TAR. This success comes despite a mean antegrade cerebral perfusion duration of 2 hours. The in-hospital mortalities of $14.7 \%$ in the non-TAR group and $10.2 \%$ in the TAR group demonstrate the high acuity and risks of these patients with acute dissections. Not surprisingly, multivariate analysis revealed that preoperative cardiopulmonary resuscitation and end-organ malperfusion are significant predictors of mortality. Interestingly, TAR was not associated with poor outcome. Furthermore, 5-year survival was not statistically different between the TAR and non-TAR groups. The rate of distal aortic events at 5 years, however, was significant improved in the TAR group. Omura and colleagues ${ }^{1}$ concluded that operative mortality of patients with acute DeBakey I aortic dissection treated by TAR was acceptable with good long-term outcome.

Although the conclusions of the article are reasonable, this is a moderately sized single-institutional, retrospective, observational study that warrants caveats in its interpretation. Selection bias remains likely in the determination of which patients undergo TAR versus non-TAR and surely includes selection factors in addition to the location of the dissection tear. Although there was no statistical significance, the in-hospital and 30-day mortalities were higher in the non-TAR group. This is most likely a consequence of a higher proportion of non-TAR patients

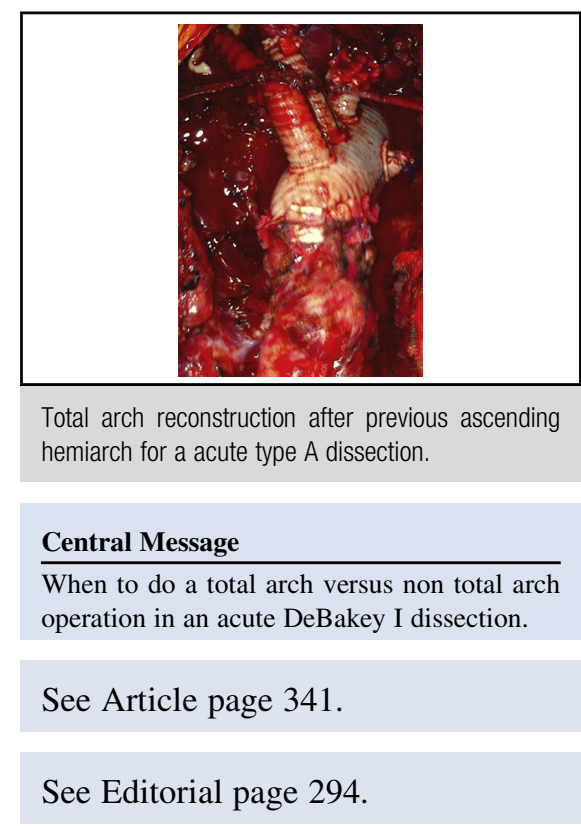

who were seen in cardiogenic shock. Furthermore, patients undergoing non-TAR were more likely to be older, to require cardiopulmonary resuscitation, and to have a larger distal aortic diameter. Despite the tear-oriented paradigm, $19 \%$ of patients who underwent TAR had a tear in the ascending aorta only, which did not adhere to their treatment paradigm and suggests that a more aggressive approach was taken in some cases because of additional selection factors.

With regard to distal aortic remodeling, Omura and colleagues ${ }^{1}$ found that the freedom from distal aortic events was worse in patients undergoing non-TAR. This difference may have been influenced, however, by the finding that patients undergoing non-TAR were more likely to have false-lumen patency, as well as a larger diameter at the level of the distal arch during the initial time of presentation and time of the operation. In contrast, it is more interesting that the freedom from distal aortic intervention and long-term survival at 10 years were similar between TAR and non-TAR groups. The argument can be made that these long-term end points are the objectives of the surgical therapy for dissections and therefore more clinically relevant.

Despite the caveats, the study does provide several important messages. First and foremost, it is of utmost importance to perform the "appropriate operation" on the basis of the presenting clinical scenario and appropriate 
patient selection. Decision making in reconstruction of the aortic arch in acute DeBakey I aortic dissection must reflect a rational balance between an extensive reconstruction that addresses the pathology (ie, the dissection tear) and a recognition of higher mortality associated with increasing surgical complexity. Surgical concepts, including this tearoriented paradigm in aortic dissection repair, should always be viewed as guidelines rather than surgical dogma that cannot be deviated from or violated. We do believe that frail, older patients with significant comorbidities who are seen with hemodynamic collapse are likely best served with a less-aggressive non-TAR procedure when this is technically reasonable (the "less is more" approach). This is particularly acceptable and important because of the long-term survival results published by Omura and colleagues. ${ }^{1}$ An aggressive tear-oriented approach to perform a TAR operation when the tear involves the arch, however, should be seriously considered when appropriate, particu- larly in younger patients with connective tissue disorders. Similar to other studies, ${ }^{2,3}$ this study demonstrates that TAR for acute DeBakey I aortic dissection can be performed safely with acceptable outcomes in aortic centers of excellence. Finally, this study further strengthens the importance of a comprehensive, experienced aortic team in the management of these high-risk patients.

\section{References}

1. Omura A, Miyahara S, Yamanaka K, Sakamoto T, Matsumori M, Okada K, et al. Early and late outcomes of repaired acute DeBakey type I aortic dissection after graft replacement. J Thorac Cardiovasc Surg. 2016;151:341-8.

2. Rylski B, Milewski RK, Bavaria JE, Vallabhajosyula P, Moser W, Szeto WY, et al. Long-term results of aggressive hemiarch in 534 patients with type A aortic dissection. J Thorac Cardiovasc Surg. 2014;148:2981-5.

3. Shi E, Gu T, Yu Y, Yu L, Wang C, Fang Q, et al. Early and midterm outcomes of hemiarch replacement combined with stented elephant trunk in the management of acute DeBakey type I aortic dissection: comparison with total arch replacement. J Thorac Cardiovasc Surg. 2014;148:2125-31. 